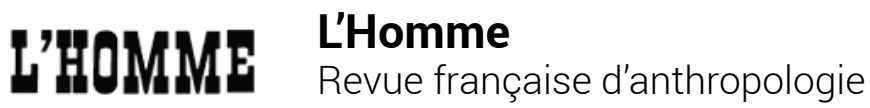

Oralité et écriture

\section{Sally Price, Paris Primitive. Jacques Chirac's Museum on the Quai Branly}

\section{Fabrice Grognet}

\section{(2) OpenEdition \\ 1 Journals}

\section{Édition électronique}

URL : http://journals.openedition.org//homme/28780

DOI : 10.4000//homme.28780

ISSN : 1953-8103

Éditeur

Éditions de l'EHESS

\section{Édition imprimée}

Date de publication : 1 janvier 2009

Pagination : 280-282

ISSN : 0439-4216

\section{Référence électronique}

Fabrice Grognet, "Sally Price, Paris Primitive. Jacques Chirac's Museum on the Quai Branly», L'Homme

[En ligne], 189 | 2009, mis en ligne le 03 janvier 2017, consulté le 24 septembre 2020. URL : http:// journals.openedition.org/lhomme/28780 ; DOI : https://doi.org/10.4000/lhomme.28780

Ce document a été généré automatiquement le 24 septembre 2020.

(c) École des hautes études en sciences sociales 


\title{
Sally Price, Paris Primitive. Jacques Chirac's Museum on the Quai Branly
}

\author{
Fabrice Grognet
}

\section{RÉFÉRENCE}

Sally PRICE, Paris Primitive. Jacques Chirac's Museum on the Quai Branly, Chicago-London, University of Chicago Press, 2007, 239 p., bibl.

1 LE NOUVEAU LIVRE proposé par Sally Price et écrit, comme les Français se plaisent à le dire, «dans la langue de Shakespeare » (p. 180), a pour sujet central le musée du quai Branly (мQВ). Il constitue, comme le précise également d'emblée son auteure, une des nombreuses histoires possibles de la relation qu'entretient la France avec ces arts que l'on nommait autrefois « nègres » ou « primitifs » et que les convenances actuelles nous amènent à qualifier de "premiers». En ce sens, il prolonge l'analyse de la relation ambiguë entre les «arts primitifs » et les «regards civilisés» occidentaux déjà développée par Sally Price ${ }^{1}$ et rééditée dernièrement.

2 Après avoir énuméré en introduction quelles pouvaient être les autres dates historiques (depuis le musée Dauphin de 1830 jusqu'à l'exposition d'arts indiens vue par Claude Lévi-Strauss en 1943, à l'American Museum of Natural History de New York) de cette histoire mêlant politique, vie intellectuelle et monde des arts, l'auteure assume son parti pris de prendre comme objet d'analyse anthropologique ce "musée de Jacques Chirac au quai Branly » qui restera comme « l'héritage » de sa présidence.

3 Les cinq chapitres principaux reviennent sur: la rencontre aussi improbable que mystérieuse des deux «Jacques ", Chirac et Kerchache (chap. I) ; le rôle donné aux arts et en particulier au Louvre dans l'espace culturel français emprunt d'universalisme (chap. II) ; l'arrivée délicate des anciens objets ethnographiques parmi les chefsd'œuvre du Louvre (chap. III) ; les conséquences du projet de valorisation des « arts premiers" - au Louvre et au quai Branly - qui occasionne un jeu de «chaises musicales» dont l'issue est un remaniement profond des institutions culturelles 
françaises (chap. IV) perçu comme une "absolute absurdity» (p. 109); l'élaboration (laborieuse et conflictuelle en interne) du musée du quai Branly et l'analyse de ses partis pris muséographiques (chap. V). La conclusion, en forme d'« épilogue ", revient, quant à elle, sur le but premier revendiqué par le nouveau musée : le MQB met-il en dialogue les cultures?

4 A priori, on se dit que l'on connaît déjà l'histoire de la genèse de ce nouvel établissement parisien qui a suscité tant de polémiques et de publications aussi bien en France qu'à l'étranger. En 1990, Jacques Chirac, alors maire de Paris et passionné en secret d'arts lointains, rencontre, sur une plage de l'île Maurice, Jacques Kerchache, ancien galeriste controversé, sorte d'« Indiana Jones » à l'allure à la « Gainsbourg » (p. 2). À ce moment, l'ambition de Kerchache de voir le Louvre accueillir les objets qu'il nomme «d'arts premiers » (bien que l'on cherche toujours à savoir ce qui les unit sous ce terme au-delà $\mathrm{du}$ fait qu'ils appartiennent à d'autres cultures) semble être un «rêve». Les conservateurs du Louvre s'y opposent et les prérogatives des musées parisiens sont établies entre la valorisation esthétique des arts africains et océaniens Porte Dorée et la présentation ethnographique au Trocadéro. Pourtant, dix ans plus tard, "the unthinkable has happened ». Le 13 avril 2000, Jacques Chirac, entre-temps élu (en 1995) président de la République française, inaugure une section du Louvre conçue par celui qui est devenu son ami, Jacques Kerchache. Ce dernier est également l'éminence grise d'un projet présidentiel de nouveau musée concentrant au quai Branly les collections du défunt musée des Arts africains et océaniens et celles (ethnographiques) du Musée de l'Homme. Malgré la " tempête » (p. 68) soulevée par l'achat de pièces nok apparues illégalement sur le marché de l'art, les questions éthiques que soulèvent la politique d'acquisition de l'institution nationale française et le "mouvement de résistance " (p. 88) des employés du Trocadéro, le MQB se concrétise.

5 Mais ce nouvel ouvrage signé par Sally Price n'est ni une "biographie autorisée » (p. 180) ni un nouveau pamphlet destiné à dénoncer le «scandale » du musée du quai Branly à la manière de ce qu'avait livré Bernard Dupaigne, ancien directeur revanchard du laboratoire d'ethnologie du Musée de l'Homme. Dans une postface appelée «An American in Paris ", l'auteure, une des anthropologues américaines les plus françaises, montre son "affection» (p.179) toujours vivace pour la France et précise que son ouvrage doit être pris pour une «critique amicale» (p.180) de la part d'une observatrice qui n'a pas de compte à régler.

6 Si le discours des quatre premiers chapitres est essentiellement fondé sur une revue de presse qui a déjà suscité nombre d'articles et la matière première d'un autre récent ouvrage autour de l'avènement du quai Branly ${ }^{2}$, le ton, souvent teinté d'humour, et les anecdotes rapportées par les confrères et connaissances de l'auteure impliqués dans le projet, permettent de nous replonger sans peine dans une histoire dont on connaît déjà la fin.

7 Le cinquième chapitre, "An anti-palace on the Seine », est sûrement celui qui apporte toute l'originalité du présent ouvrage. Fruit des observations glanées (à partir de septembre 2005) par Sally Price au cours de ses visites du chantier auxquelles font suite ses analyses de la muséographie lors de l'ouverture (20 juin 2006), il est le résultat d'une véritable enquête de terrain qui fait renouer la démarche avec l'entreprise ethnographique. Les rencontres - parfois déconcertantes (notamment avec André Delpuech, responsable des collections "Amériques", ou encore avec Séverine Le Guével, directrice des relations internationales du musée) - avec les principaux acteurs 
du musée sont souvent l'occasion de souligner les lacunes du projet, l'emprise de l'architecte Jean Nouvel, mais aussi de faire ressortir les fondements idéologiques qui ont présidé à l'élaboration. Au nom d'une esthétisation voulant rompre avec le « temps du mépris " colonialiste, auquel le discours ethnologique semble toujours associé, les objets ne sont pas interprétés à la lumière de ce que pourraient en dire les scientifiques $^{3}$. Mais ils le sont encore moins d'après la consultation des peuples et communautés actuels dont ils sont issus en vertu du principe de laïcité des musées français mis en avant par les responsables du quai Branly (au contraire des musées anglo-saxons où les expositions sont réalisées en partenariat avec les représentants des communautés "autochtones" instaurant ainsi un dialogue autour d'un patrimoine commun). En fait, comme le souligne Sally Price présente en France lors de la flambée de violence dans les banlieues (où la religion musulmane est montrée du doigt par certains analystes) concomitante de l'entrée des Arts islamiques au Louvre, le refus de la France d'affronter les fantômes de son passé colonial et la mise en avant de son principe de laïcité semblent mettre le musée national chargé de célébrer la diversité culturelle dans la situation de "purifier» (p.128) ce qui pose problème au modèle républicain craignant un communautarisme à l'américaine. Mais à l'« harmonie » toute apparente du monde des musées correspondent les troubles bien réels d'une société devenue multiculturelle.

8 En définitive, comment ne pas être - au moins - dubitatif (à l'image de Kofi Annan devant la sculpture "pré-Dogon » qui inaugure la visite du "plateau des collections ») vis-à-vis de l'intérêt pour la France de s'être doté (à grands frais) d'un tel nouveau musée? Lieu "où les cultures dialoguent» seulement au niveau des "relations diplomatiques d'État à État " (p.177) en vertu du principe républicain de laïcité, le musée du quai Branly, dont le discours semble se limiter pour l'instant à une reconnaissance esthétique et ethnocentrique d'une vision fantasmée de l'altérité, semble être une exception française dans le monde des musées de civilisations. Pourtant, un de ses prochains défis ne serait-il pas d'instaurer le véritable dialogue, tant annoncé et toujours attendu, désiré par les scientifiques, confrontant l'histoire de cette ancienne puissance coloniale qu'est la France au discours des populations actuelles dont sont originaires les objets présentés? La balle est désormais entre les mains de la classe politique dirigeante.

\section{NOTES}

1. Cf. Arts primitifs, regards civilisés, Paris, École nationale supérieure des Beaux-Arts, 1995 [rééd. 2006].

2. Benoît de L'Estoile, Le Goût des autres. De l'Exposition coloniale aux Arts premiers, Paris, Flammarion, 2007.

3. Comme le montre notamment le comparatif entre le traitement historique proposé par le MQB qui expose les statues des rois Glélé et Gbéhanzin en édulcorant le contexte colonial et celui fourni par le site internet du Metropolitan Museum de New York.

4. Photographie placée en préambule du texte du livre. 


\section{AUTEURS}

\section{FABRICE GROGNET}

Cité nationale de l'immigration, Palais de la Porte Dorée, Paris.

fabrice.grognet@histoire-immigration.fr 